\section{COARSE STONE}

Rob Engl

\subsection{Introduction}

The excavation at East Barns produced a small assemblage of 21 coarse stone artefacts, all but one of which was associated with stratified deposits. Fourteen of these pieces were categorised as bevelended pebble tools. The remainder of the assemblage consisted of two hammer-stones, a knapping stone, an anvil, a burnisher and an anvil or knocking stone. An un-worked packing stone of quartzite was also included due to the nature of the raw material and its context.

The artefacts were grouped according to general characteristics such as morphology, use-wear and probable function. A detailed description of each individual artefact is given in the category sections given below.

\subsection{Raw materials}

The site is situated within till deposits overlying a solid geology of carboniferous sedimentary rocks (Bown \& Shipley 1982). All the artefacts are made on locally derived cobbles of water-worn sandstone, quartzite and fine-grained sedimentary rocks. These were brought onto the site from the shoreline or nearby riverine sources.
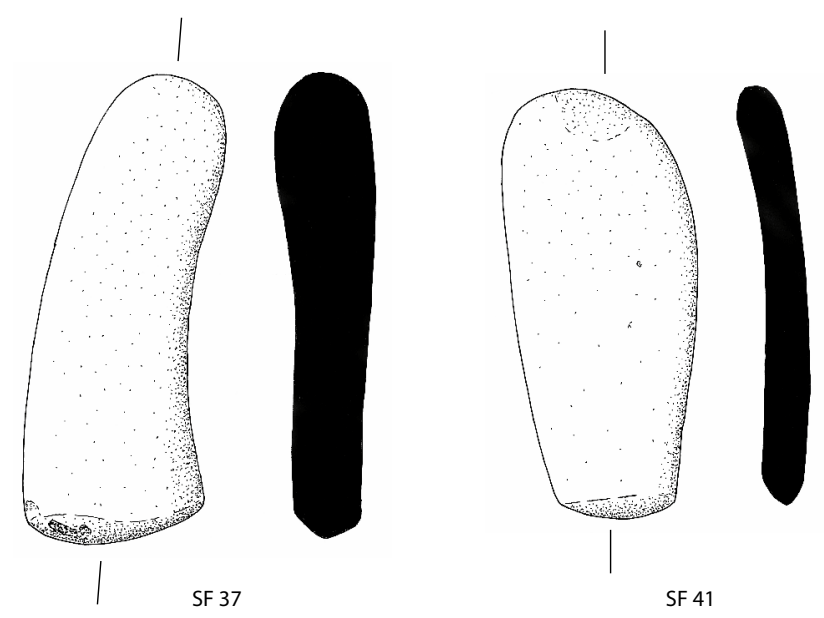

\subsection{Bevel-ended pebbles}

The excavation recovered 14 bevel-ended stone tools, all of which are made on elongated, waterworn pebbles of sandstone and fine-grained sedimentary rock. Nine of the pieces were complete and five were fragmentary. The size of the complete pieces varies but all are at least twice as long as they are wide. A selection of the tools is illustrated in Illus 20 and 21.

All of the pieces show evidence of use occurring in the form of a bevel, probably formed through a grinding or rubbing action of a rounded end of the cobble. In 11 of the pieces the bevel is bifacial in character, with the remainder unifacial. Only one example has bevelling at both ends of the cobble. SF 38 has a bevel that shows surface damage in the form of pitting and flake scars (Illus 21). On all pieces the bevel is generally pronounced, with the exception of SF 44, on which only a lightly formed area of wear is visible.

It is not clear whether the fragmentary pieces were broken during use or became fractured after abandonment. However, the fragmentation pattern is similar to those occurring in other Mesolithic assemblages and from experimental examples broken during use (Barlow \& Mithen 2000: 517).
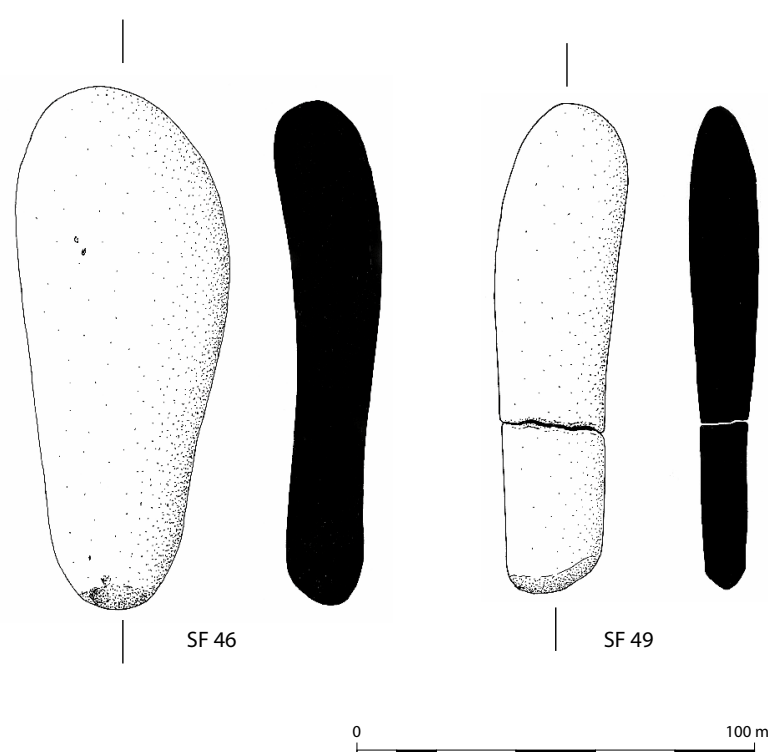

$100 \mathrm{~mm}$

Illus 20 Bevel-ended pebbles 

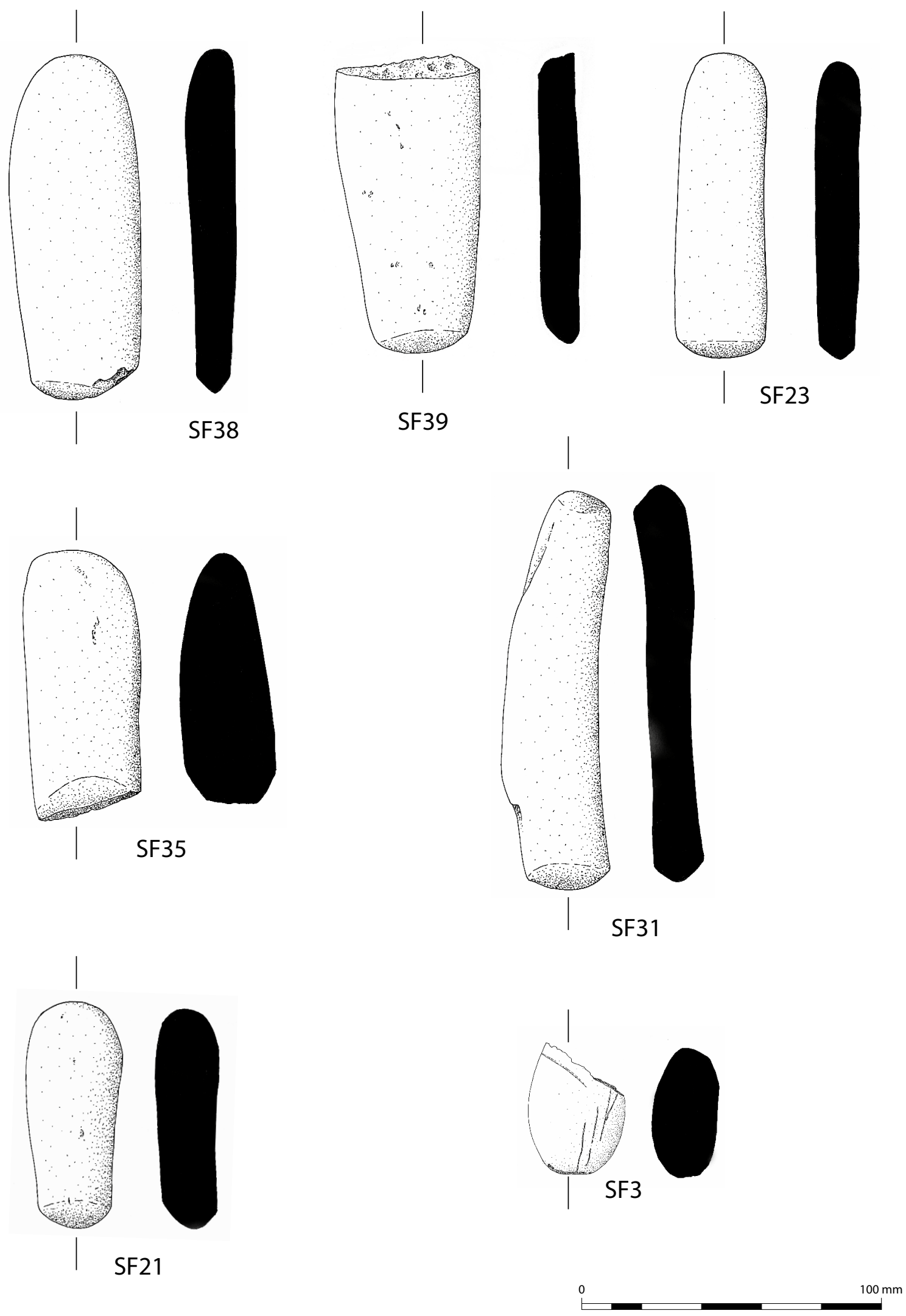

Illus 21 Bevel-ended pebbles and coarse stone tools (SF 03 and SF 21) 


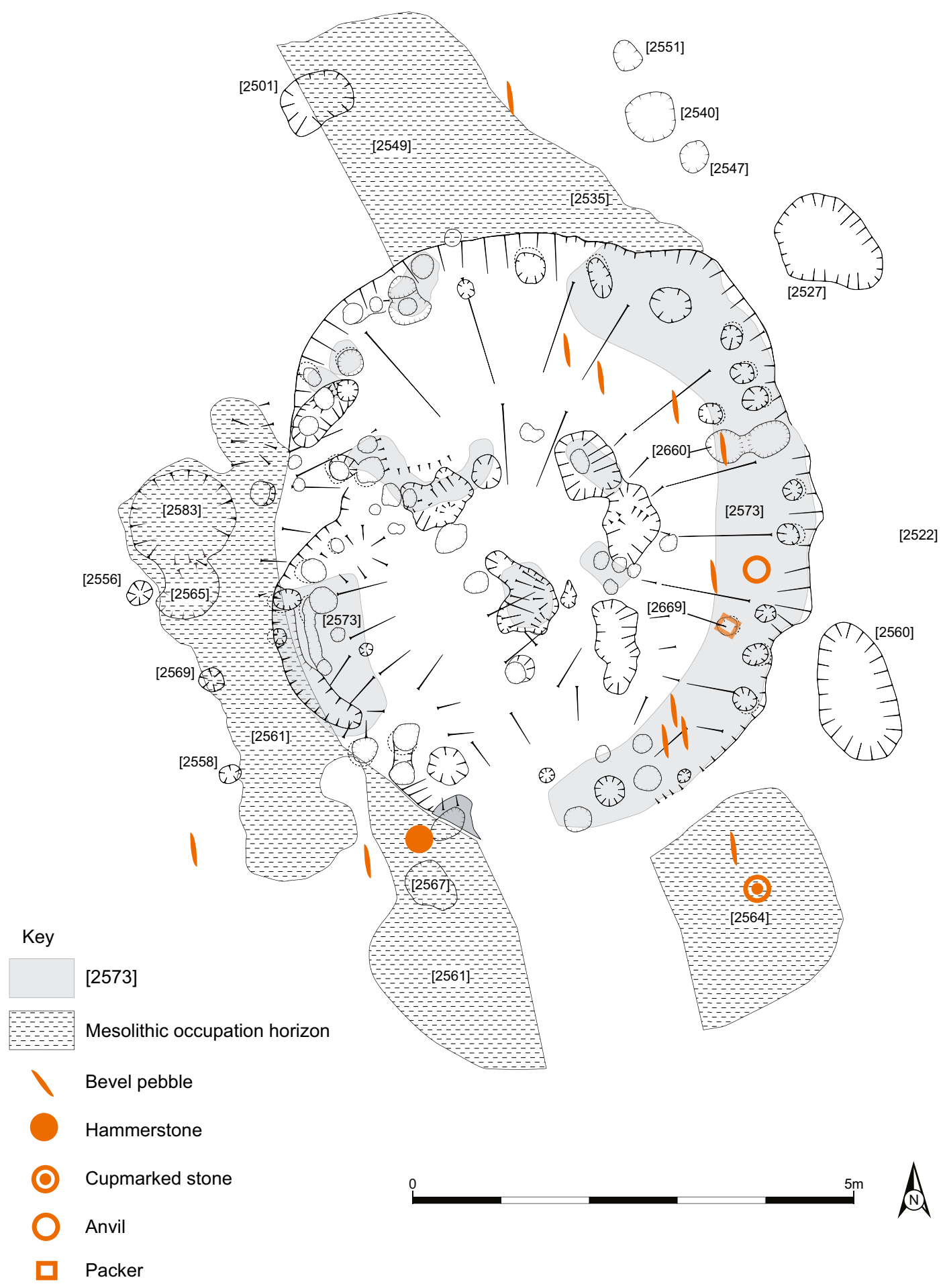




\subsubsection{Distribution (Illus 22)}

All of the bevel-ended tools were recovered from stratified contexts associated with the prehistoric occupation of the hollow (2522).

Six of the artefacts were directly associated with the Mesolithic house. These included two pieces identified as packing stones recovered from within post hole features (SF 41 and 31). Four pieces were also retrieved from occupation layers both within and surrounding the house structure. Three of these pieces (SF 37, 38, 39) were retrieved from the in situ deposit (2573) located within the interior of the house. A solitary piece (SF 32) was also recovered from the exterior activity area to the immediate south-west of the house.

Eight bevel-ended tools were also associated with the colluvium infilling the hollow. Four pieces were found within contexts directly overlying the house structure. A further three examples were recovered from along the inner north-eastern edge (SF 47, 48, 49) and one at the south-eastern edge (SF 44). These pieces represent material from the surrounding occupation areas washed or deliberately deposited into the structure after its abandonment. Three pieces were also recovered from areas close to the structure. SF 21 was found bordering the occupation deposit (2535), and two (SF 46 and 23) were retrieved from the south-west. A single example (SF 36) was recorded away from the immediate environs of the structure. This piece was recovered from the colluvium infilling the north of the hollow and lying close to the radiocarbon-dated Late Mesolithic deposit (2531).

Catalogue of illustrated artefacts (dimensions in $\mathrm{mm}$; weight in $g$ )

- SF 21 (2533) E15 SE Bevel-ended tool (76.2 × $33.6 \times 22.7)$ 89.18g. Complete.

Bifacial bevel on narrow end of small elongated sandstone pebble. (Illus 21)

- SF 23 (2534) B7 NW Bevel-ended tool (101.6 $\times 33 \times 14.7)$ 95.8g. Complete.

Bifacial bevel on wider end of sub-rectangular sandstone pebble. (Illus 21)
- SF 31 (2561) Bevel-ended tool (133.8 × $35 \times$ 15.7) 143.5g. Complete.

Double ended with one end bifacial and one unifacial, on elongated oval pebble of fine-grained sedimentary rock. (Illus 21 )

\section{- SF 37 (2573) E8 SE Bevel-ended tool (122.2 ×} $45.6 \times 24.8) 125$ g. Complete.

Bifacial bevel on single end of elongated, oval pebble of sedimentary rock. (Illus 20)

\section{- SF 38 (2573) E8 SE Bevel-ended tool (114.6 ×} $44.6 \times 18) 165.9$ g. Complete.

Bifacial bevel with some pitting on elongated oval pebble of fine-grained sedimentary rock. (Illus 21)

\section{- SF 39 (2573) E8 SE Bevel-ended tool (96.3 ×} $50.6 \times 16.7) 125 \mathrm{~g}$. Fragment.

Bifacial bevel on single end of elongated, oval sandstone pebble. (Illus 21)

\section{- SF 41 (2628) Bevel-ended tool (109.8 $\times 47.1 x$ 12.8) 120.9g. Complete.}

Bifacial bevel on narrow end of flat, elongated sandstone pebble. (Illus 20)

\section{- SF 46 (2553) Z8 SE Bevel-ended tool (130.6 ×} $53.5 \times 27.3)$ 292.6g. Complete.

Unifacial bevel on single end of elongated, flat, sandstone pebble. (Illus 20)

- SF 49 (2550) E12 NE Bevel-ended tool (123.4 $\times 31.6 \times 17.7)$ 88.04g. Fragment

Bifacial bevel on narrow end of flat, elongated sandstone pebble. (Illus 20)

\subsection{Other coarse stone}

Six other tool types make up the remainder of the coarse stone assemblage. These consist of a small knapping stone (SF 3), two cobble hammer-stones (SF 43), a burnisher (SF 35), an anvil (SF 25), a large packing stone and an anvil/knocking stone (SF 42) (Illus 21 and 22). This latter piece is made on a large roughly oval, flat surfaced cobble of fine-grained sedimentary rock. It has a circular, roughly pecked indentation with peck marks also scattered across the surface. It is likely that this piece represents an anvil used for the initial reduction of lithic material 
or perhaps even for the cracking of hazelnuts or other hard-shelled foodstuffs.

The small knapping stone (SF 3) is made on a small water-worn quartz pebble with dense percussion wear on one end. This artefact is much smaller than the hammer-stones so it is likely that this artefact would be used for more precise tasks, such as tool production or modification requiring more general control and dexterity.

\subsubsection{Distribution (IIlus 22)}

Of the seven pieces of coarse stone tools, three were associated with the infilling deposit (C2550). The knapping stone SF 3 was also associated with a deposit (C2521) infilling the house structure. The quartzite packing stone was retrieved from structural Post Hole 2669. The anvil/knocking stone SF 42 was recovered from the occupation deposit (C2564) located to the immediate south-west of the structure.
Catalogue of illustrated artefacts (dimensions in $\mathrm{mm}$; weight in $g$ )

- SF 3 (2521) Knapping stone $(39 \times 30.5 \times 26.4)$ 49.9g. Complete.

Small hammerstone on water-worn quartz pebble. One end has severe percussion wear. (Illus 21)

\section{- SF 25 (2550) G9 NW Anvil (88.7 × $45.2 \times 31.4)$ 163.2g. Complete.}

Rounded sandstone cobble with flattish faces. Centralised wear on one face in the form of pecking. (Illus 23)

- SF 35 (2550) Burnisher (145.1 × $74.8 \times 52)$ 900.6g. Complete.

Fine-grained sedimentary cobble with single smoothed area on one face. Wear extends slightly onto one edge. (Illus 21)
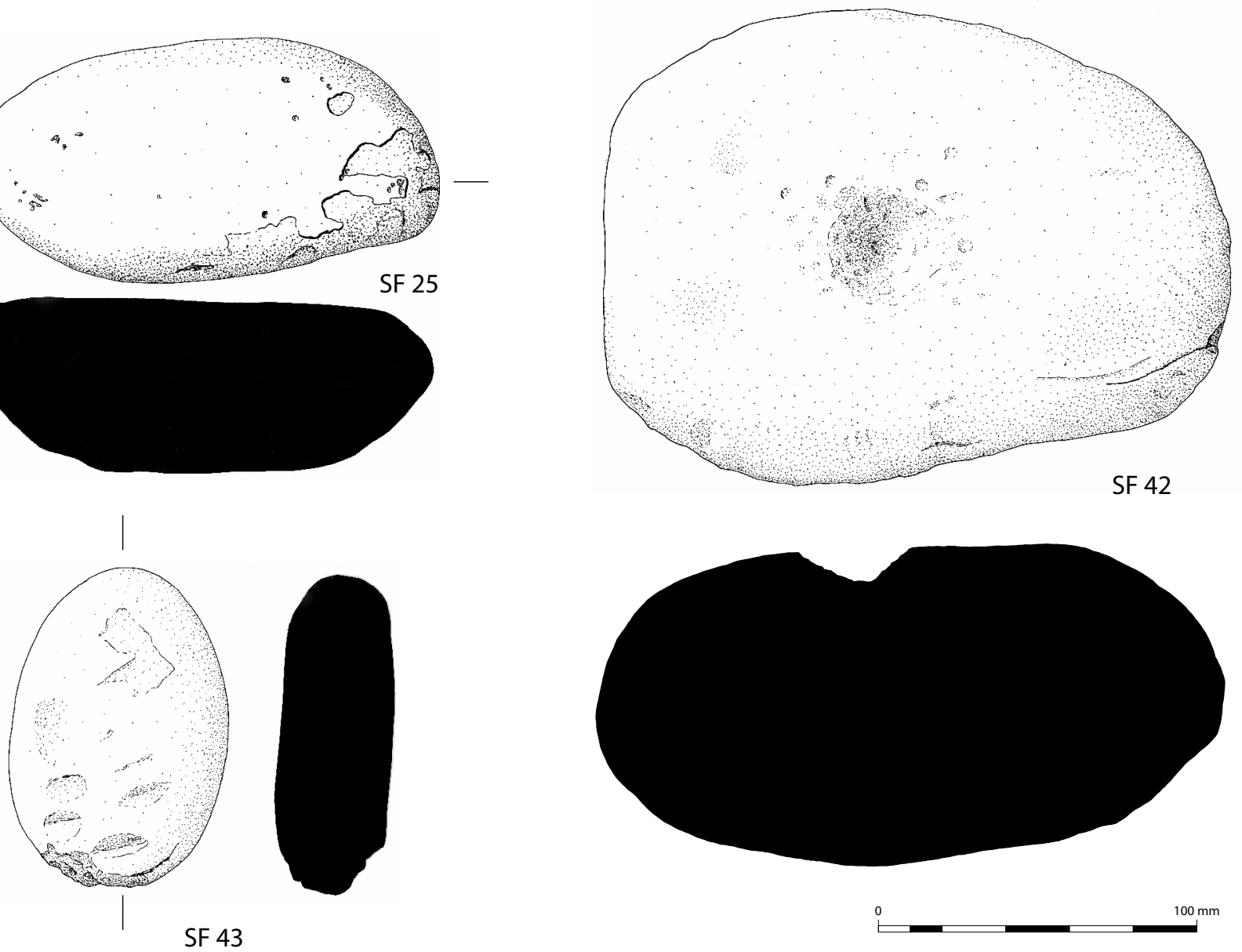

Illus 23 Other coarse stone tools 
- SF 42 (2564) Anvil or knocking stone (190 × $140 \times 85)$. Complete.

Large cobble of fine-grained sedimentary rock with centrally placed pecked circular indentation measuring $38.6 \times 38.6$. The indentation has a depth of $13.2 \mathrm{~mm}$. Peckmarks are also scattered across the surface. (Illus 23)

\section{- SF 43 (2550) B7 NE Hammerstone (100.4 $\times$ 68.5 × 36.2). 303.6g. Complete.}

Cobble of foliated sandstone with severe damage along one edge. (Illus 23)

\subsection{Discussion}

Coarse stone artefacts form an important though often ignored source of evidence for reconstructing Mesolithic culture. Though often small in number, a recurring range of tools is associated with both microlithic and non-microlithic Mesolithic sites. The bevel-ended cobble tools, hammer-stones and anvils present at East Barns therefore form a recognisable set of artefacts that have parallels in assemblages throughout northern Britain. Together with the assemblage at Howick (Waddington 2007), East Barns provides a well-stratified source of information away from the heavily studied sites of the Atlantic seaboard.

Bevel-ended stone tools form the mainstay of the coarse stone assemblages of both East Barns and Howick. Such tools made on stone, antler and bone are found throughout Britain, Ireland and Brittany (Warren 2005: 100). The early dates coming from these two sites mean that bevel-ended tools are now known throughout the Mesolithic period of Scotland and beyond.

Within the Mesolithic, these tools have a primary association with coastal midden and Obanian cave sites (Anderson 1898; Bishop 1914; Coles 1971; Mellars 1987; Saville 2004: 191). Examples of bone and antler tools are almost solely restricted to midden sites where conditions of preservation are favourable, whereas quantities made on stone have been found on narrow-blade microlithic sites such as East Barns, Kinloch Farm, Rhum (Clarke 1990: 120), Howick (Waddington 2007) and Camas Daraich (Clarke 2004: 46).

A coastal or near-coastal location appears to link all of the assemblages in which bevel-ended tools are found. David \& Walker (2004: 323) have stated that these artefacts are a product of rocky coastlines, particularly along the Atlantic seaboard. The sites of Howick and East Barns now lie close to the shoreline, but whether they were of a similar rocky appearance during the site's occupation is open to conjecture.

The apparent restriction of bevel-ended tools to coastal or near-coastal sites would initially suggest a role in exploiting a particular set of marine resources. Bevel-ended tools of all materials were originally interpreted as limpet hammers (Grieve 1885: 57) or limpet scoops (Bishop 1914: 95). Although this interpretation has been heavily criticised (Finlayson 1995), experimental work has shown that bevel-ended cobble tools could successfully be used to remove limpets (Barlow \& Mithen 2000; Birch 2009), with the action producing similar abrasion and breakage patterns to those identified within the assemblages at East Barns, Howick and other Mesolithic sites.

Other proposed functions have included flint knapping (Breuil 1922: 267-71; Saville 2004: 191), while the experiments on bone bevel-ended tools undertaken at Sand as part of the Scotland's First Settlers Project (Birch 2009: 293) proved that these tools could undertake a variety of other functions, including plant processing, bark removal and hide working.

The latter was also proposed by Foxon (1991), Finlayson (1995; 1998) and Griffiths \& Bonsall (2001) on stone bevel-ended tools. The possible use in hide working was first addressed by Anderson (1895: 222), who thought that the more common smaller bevel-edged tools found in middens were likely used for the dressing of hides. Jacobi (1980: 189) has in turn associated bevel-edged tools with the dressing of seal skins, an attractive theory given the general locations in which these artefacts are found.

Finlayson (1995: 262) argues that the identification of these artefacts as limpet scoops ignores the lack of shell midden associations found away from the west coast. This is very much the case at sites such as East Barns, Howick and Kinloch Farm, where bevel-ended tools were not found in close association with sources of shellfish, the likelihood being that the sites were situated some distance from the Mesolithic coastline during their occupation. 
Though named after the characteristic wear created by their use, one must keep in mind that the natural rounded edge of the cobble tool was the desired working edge. This edge is common to water-worn cobbles of all shapes and sizes and would in most cases be more than capable of removing shellfish. Therefore, the need for a dedicated, elongated cobble tool is hard to justify.

The distributions of these artefacts at East Barns suggest a close association with tasks undertaken in and around the house. Three were found in the internal detritus deposit (2573), while another two examples were re-used as post hole packing stones. This suggests that the pieces may have been used in domestic tasks within the house itself. Those recovered from in and around the outer occupation deposits may reflect direct use in these areas or may represent dumped material from the inside of the structure. The artefacts from the infilling colluvium may also represent material washed or thrown into the house area after abandonment.

The elongated shape and presence of a bevel are unifying morphological factors when discussing these artefacts in all materials, and imply a common function. Warren (2005: 100) however, notes that the physical properties of bevel-ended tools, whether made on stone, bone or antler, are very different and that whatever use(s) these tools were put to, the choice of material was deliberate, perhaps reflecting differing functions.

The deliberate choice of materials may also be reflected in the general size range of these implements. The mean dimensions of the complete tools recovered from East Barns were compared with samples from the near-coastal narrow-blade microlithic sites of Howick, Kinloch and Staosnaig. These were then compared with the samples given in Finlayson (1995: 262), which included mixed stone and bone tools recovered from five largely non-microlithic (with the exception of Morton) coastal midden and cave sites (Table 16).

A large discrepancy in size was observed, with those stone tools recorded from the near-coastal microlithic sites being almost twice as long as those examples obtained from the coastal cave and midden samples. While this may be a result of geological circumstance, it could represent a deliberate selection of material. This in turn may reflect the possibility of functional differences. It is therefore possible that smaller bevel-ended tools of both bone and stone were used to exploit marine resources and as such were abandoned near their place of use in coastal caves and middens. The larger pieces, made on stone, were possibly used in base camp activities such as the dressing of hides or flint-working at sites situated some distance from the foreshore.

This deliberate choice of materials may also have a chronological aspect. The four occupation sites associated with narrow-blade microliths all produced relatively early radiocarbon dates. These ranged from $c 7800 \mathrm{cal} \mathrm{BC} \mathrm{(Howick)} \mathrm{and} c 8000 \mathrm{cal}$ BC (East Barns and Kinloch Farm) to $7000 \mathrm{cal} \mathrm{BC}$ at Staosnaig. With the exception of Morton, which is associated with a broad-blade microlithic industry, coastal midden and cave sites produced a uniformly later Mesolithic range of dates.

Unlike the other sites mentioned above, Morton is considered to represent repeated low-level

Table 16 Coarse stone: metrical comparison of bevel-ended pieces (mean values)

\begin{tabular}{lcccc} 
& Number studied & Length & Width & Thickness \\
East Barns & 9 & 111.3 & 40.4 & 20 \\
\hline Kinloch Farm & 9 & 99 & 40.4 & 20.1 \\
\hline Staosnaig & 9 & 112.5 & 19.2 & 12.2 \\
\hline Carding Mill Bay & $?$ & 45.3 & 13.5 & 7.3 \\
\hline MacArthur's Cave & $?$ & 54.7 & 15.9 & 8.8 \\
\hline Cnoc Sligeach & $?$ & 58.7 & 18.4 & 11 \\
\hline Morton & $?$ & 60.7 & 18.6 & 8.9 \\
\hline Cnoc Reach & $?$ & 67.4 & 16.2 & 11.6 \\
\hline
\end{tabular}


transitory occupation by small numbers of people. The bevel-ended tools recovered from the midden at this site are not considered morphologically similar to those from the west coast (Finlayson 1995: 262), and no bevel-ended stone cobble tools were excavated. This is reversed at both Howick and East Barns. It is presumed that such bone tools are absent on these sites due to a simple lack of survival. This is a problem common to many non-midden sites in Scotland.

Stone bevel-ended tools do not occur on all Mesolithic occupation sites with structural evidence. At Mount Sandel, Northern Ireland (Woodman 1985) and the Mesolithic structures excavated at Echline Fields (Robertson et al 2013), no examples of bevel-ended cobble tools were recovered from the excavations of the structures. This was also the case at Newton, Islay (McCullagh 1989), where the excavation of a large hollow, thought to be the base of a Mesolithic structure, produced no coarse stone tools. While recognising the possibility that artefacts of this type were overlooked during excavation, it is likely that these locations represent sites such as processing or long-term hunting camps where the range of activities did not require the use of certain tools.

Of the other tool categories present at East Barns, parallels can be drawn with many other Mesolithic and later prehistoric sites. As Saville notes, 'few coarse stone tools are reliably diagnostic, being a continuing facet of tool use in Scotland well into the first millennium AD' (Saville 2003; 2005: 191). Fortunately, East Barns saw a close association between these tools and both Mesolithic cultural material, and dated deposits.
The hammer-stones, anvil and knapping stone are most obviously associated with the on-site reduction of lithic material. The stone with the pecked hollow recovered from the occupation deposit (2564) is similar in form to the hollowed stone from Fife Ness (Wickham-Jones \& Dalland 1998, illus 9). No specific function was assigned to this artefact, although the possibility of use as an anvil was noted. The artefact from East Barns differs in that it is larger and the hollow is deeper and formed by pitting. Scattered elements of pitting are also visible across the face of the piece. This would suggest intensive use as an anvil for lithic reduction.

Despite the presence of later prehistoric material at East Barns, the coarse stone artefacts can confidently be associated with activities undertaken in and around the Mesolithic house. As is often the case, a more precise picture of the roles these tools played in Mesolithic life is difficult to assess. The presence of hammer-stones and anvils in association with large quantities of lithic material would support a primary role in lithic reduction. However, as with many types of coarse stone tools, their use in a variety of other activities, such as food processing, cannot be discounted. Similarly, in view of the still-conflicting experimental evidence for the use of bevelled pebbles, their use as general purpose tools remains the most plausible hypothesis. At East Barns, both artefact distribution and site location suggest a use in hide dressing, knapping or other camp-based activities.

The small coarse stone assemblage at East Barns provides a valuable, stratified source of evidence for Mesolithic culture on the east coast of Scotland. 